\title{
Caisse-maladie de la CPT
}

\section{Profitez du contrat collectif de la FMH Insurance Services}

\section{Excellente relation prix/prestations}

Affiliez-vous au contrat collectif CPT spécialement conçu pour la FMH Insurance Services. Profitez des prestations élevées et des rabais de primes importants.

\section{Votre gain}

Sur les prestations complémentaires suivantes, nous vous offrons des réductions de primes:

Frais d'hospitalisation-15\% de rabais

Prise en charge des frais d'hospitalisation au choix: division commune, division demi-privée et division privée.

Assurance des soins Plus $-10 \%$ de rabais

Prise en charge de frais supplémentaires pour des prestations en cas de traitement ambulatoire telles que médicaments, lunettes, examens préventifs, en cas de maladie aiguë ou d'accident durant un séjour temporaire à l'étranger (maximum 8 semaines), vaccinations etc.

\section{Natura $-10 \%$ de rabais}

Prise en charge des méthodes curatives alternatives auprès de médecins et de thérapeutes. En plus nous vous offrons des primes attractives pour l'assurance obligatoire des soins. Cette offre est valable pour tous les médecins membres de la FMH ainsi que les personnes membres de la famille et/ou du/de la partenaire habitant sous le même toit. Une entrée jusqu'à l'âge de 70 ans est possible. Si vous désirez être assuré auprès de la CPT contrat collectif, appelez-nous afin de vous faire profiter à l'avenir de conditions avantageuses.

\section{Profitez de notre offre}

Profitez des avantages de notre contrat collectif de la FMH Insurance Services auprès de la CPT et commandez encore aujourd'hui votre offre personnalisée en utilisant le talon réponse ci-dessous (veuillez joindre une copie de votre police actuelle pour chaque membre de la famille).

\section{Talon réponse}

Prénom / Nom

Adresse

NPA/Lieu

Date de naissance

Téléphone privé / cabinet

Atteignable le plus facilement

Prière de m'envoyer une offre. La copie de la police actuelle est jointe.

Je désire un conseil personnalisé. Veuillez m'appeler.

Je m'intéresse aux thèmes suivants:

\footnotetext{
Assurance décès

3e pilier lié et libre

Planification financière

O

Caisse de pension LPP

Protection juridique

Responsabilité civile professionnelle
}

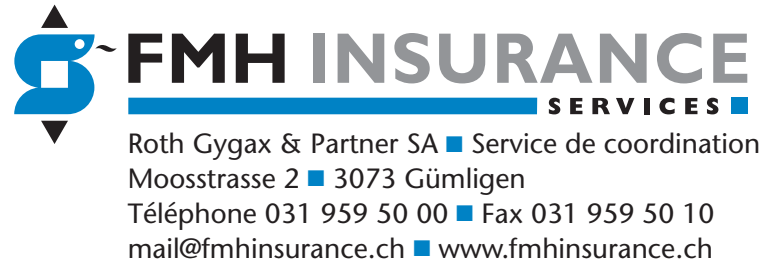

